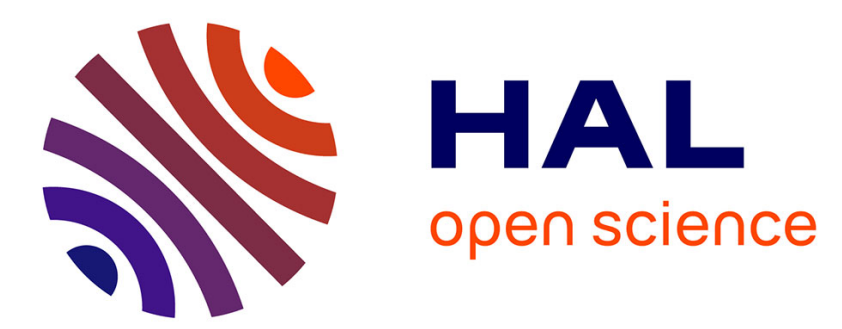

\title{
Les déterminants économiques du vote - 1976-2006 : trente ans de fonctions de vote en France
}

Eric Dubois

\section{To cite this version:}

Eric Dubois. Les déterminants économiques du vote - 1976-2006: trente ans de fonctions de vote en France. Revue d'Economie Politique, 2007, 117 (2), pp.243. hal-00800662

\section{HAL Id: hal-00800662 \\ https://hal.science/hal-00800662}

Submitted on 14 Mar 2013

HAL is a multi-disciplinary open access archive for the deposit and dissemination of scientific research documents, whether they are published or not. The documents may come from teaching and research institutions in France or abroad, or from public or private research centers.
L'archive ouverte pluridisciplinaire HAL, est destinée au dépôt et à la diffusion de documents scientifiques de niveau recherche, publiés ou non, émanant des établissements d'enseignement et de recherche français ou étrangers, des laboratoires publics ou privés. 


\title{
Les déterminants économiques du vote 1976-2006 : trente ans de fonctions de vote en France
}

\author{
Éric Dubois*
}

Résumé : cet article a pour but de présenter un panorama quasi-exhaustif de la littérature publiée ou non - sur les modèles politico-économiques explicatifs du vote en France. La définition des variables utilisées, les techniques d'estimation employées, les résultats de ces estimations et les performances prédictives des modèles sont examinés. Des faiblesses dans chacun de ces domaines sont aussi soulignées. Enfin, quelques pistes pour des recherches futures sont dégagées.

Mots clefs : économie du vote, modèle politico-économique, prévision électorale.

Classification JEL : C53, D72.

* Chercheur associé, LAboratoire d'Économie Publique, Université de Paris 1 - PanthéonSorbonne -, Maison des Sciences Économiques, 106-112, Bd. de l'Hôpital, 75647 Paris cedex 13. Tel : 33.(0)1.44.07.81.01. Eric.Dubois@univ-paris1.fr. Je remercie les participants du séminaire d'économie publique du LAEP (Paris 1), du séminaire d'économie politique d'EUREQua (Paris 1) et du séminaire de thèse du LEP/3DI (Paris 2) pour leurs commentaires et leurs apports substantiels aux premières versions du présent article. Ma gratitude va également à Patricia Vornetti pour son travail de relecture. Je remercie enfin les deux rapporteurs anonymes ainsi que le Comité de Rédaction de la revue pour leurs conseils et suggestions. 


\title{
Economic voting in France, thirty years of vote functions (1976-2006)
}

\begin{abstract}
The aim of this article is to present a very detailed survey on politico-economic models of voting built in the French case. Since 1976 and the first study ever published on this topic, more than seventy papers have been written. The $30^{\text {th }}$ anniversary is the occasion to explore this growing literature. After an introduction that highlights the main features of the scientific production and recalls the theoretical grounds of vote-functions, the first section studies the type of elections and the aggregation level in French voting models. Issues linked to the construction of the dependent variable like left-right cleavage, extreme right status, and relevant round are tackled in the second section. The third one shows the explanatory variables used. They are gathered in three groups: economic variables, institutional variables, and political variables. The conclusion regarding each of them is indicated. The section ends on the role of the campaign and on the French voter's degree of memory. A paragraph is also devoted to seats-functions that translate vote into seats. This kind of functions is very useful in majority systems like the French one because to be majority in vote does not necessary mean to be majority in seats. Section four deals with econometric problems. After having examined the way that authors treat usual diagnoses (autocorrelation, heteroskedasticity...), two remarks are drawn concerning the use of the intercept term in pooled-data models and the estimation of systems formed by a vote-equation and a seat-equation. Section five presents the forecasts produced by the models. Their accuracy is assessed by comparing the forecasted value to the actual value for some elections and by examining the forecasts stemming from vote intentions polls. This evaluation shows several brilliant successes on the recent period. The conclusion sketches some trails for future researches. Among them, we can cite the building of a finer model for the legislative elections (by constituencies for example) and the development of a model for cantonal elections. Moreover, seats-functions have to be improved and more attention has to be paid to econometrics. Taking spatial interactions into account may also constitute an appealing way to increase our knowledge of voting behaviour.
\end{abstract}

Keywords: economic voting, politico-economic model, electoral forecasting. 


\section{Introduction}

Francis Edgeworth, le célèbre statisticien et micro-économiste illustra la loi de Gauss par la répartition des écarts entre les voix reçues lors des élections et les sièges obtenus (Edgeworth [1898]). De même, Harold Hotelling, dans son célèbre article sur la localisation des entreprises, fait une analogie entre la concurrence économique des firmes et la concurrence politique des partis qui, pour gagner des voix, adoptent des positions modérées (Hotelling [1929]). Encore, Henri Theil, un des précurseurs en matière de prévision économique et inventeur, entre autres, de la technique économétrique des doubles moindres carrés et des triples moindres carrés, a étudié la relation entre sièges et votes (Theil [1970]). Et la liste pourrait être encore longue. Parmi les objets d'études politiques des économistes, figure le vote modélisé à la suite de Kramer [1971] pour les États-Unis, par une équation de vote ${ }^{1}$.

Une équation de vote est une relation statistique qui lie le vote à ses déterminants. Ces derniers peuvent être économiques, sociologiques, politiques, etc. La forme générique d'une équation de vote s'écrit :

$$
\text { vote }=\mathrm{f}(\text { facteurs économiques, facteurs politiques...) }
$$

Cette équation est à rapprocher de l'équation de popularité dans laquelle la popularité est substituée au vote (Goodhart et Bhansali [1970], Mueller [1970]).

S'il est aujourd'hui admis que l'économie influence le vote, les modalités de cette influence restent mystérieuses ${ }^{2}$. Selon Downs $[1957 \mathrm{a}]^{3}$, les citoyens agissent rationnellement en politique comme ils agissent rationnellement en économie : "chacun d'entre eux envisage les élections uniquement comme un moyen de sélectionner le gouvernement le plus avantageux pour lui. Chaque citoyen évalue l'utilité qu'il pense que chaque parti lui apporterait s'il était au pouvoir dans le futur" (Downs [1957a], page 138). Cependant, la collecte et le traitement de l'information nécessaire pour évaluer la situation future sont coûteux et conduisent l'électeur rationnel à ne pas acquérir de l'information. Selon le célèbre oxymore de Downs [1957a, page 139], l'électeur est "rationnellement ignorant". Face à ce manque d'information, les électeurs peuvent alors se contenter d'un "référendum sur la gestion

\footnotetext{
${ }^{1}$ On emploie indifféremment les expressions "d'équation de vote" et de "fonction de vote".

${ }^{2}$ Pour un bilan récent de la littérature sur ce thème, on pourra se reporter à Lewis-Beck [2005, 2006] et à LewisBeck et Stegmaier [2000, 2006].

${ }^{3}$ Les arguments sont développés extensivement dans Downs [1957b].
} 
du sortant" (Lafay [1995a], page 23) en évaluant le bilan du sortant ${ }^{4}$. Ce type de comportement, dit "rétrospectif", est également associé aux travaux de Vladimir Key qui ont consacré le vote comme mécanisme de sanction / récompense (entre autres, Key [1966]). Si les performances macroéconomiques du sortant sont satisfaisantes, l'électeur le reconduit et "maintient le statu quo politique" (Rosa et Amson [1976], page 1105) ; dans le cas contraire, il vote contre.

La théorie rétrospective, testée et vérifiée empiriquement par Kramer [1971] sur données macro et par Fiorina [1981] sur données individuelles, va se voir opposer au début des années 1980 une théorie alternative, la théorie du comportement prospectif, selon laquelle les citoyens ne sont plus tournés vers le passé mais vers le futur. $\mathrm{Si}$ des conditions économiques satisfaisantes sont anticipées pour la période post-électorale, les électeurs reconduisent le parti sortant même s'il a obtenu de mauvaises performances économiques avant les élections (Kuklinski et West [1981]).

A la fin des années 1970, un autre clivage va se faire jour. On considérait jusqu'alors, à la suite de Campbell et al. [1960, page 381], que les électeurs votaient exclusivement "selon l'état de leur portefeuille", selon l'évolution de leur situation financière individuelle (Weatherford, [1978]). Kinder et Kiewiet [1979] opposent désormais les doléances personnelles ("personnal grievances") aux jugements collectifs. Dans le premier cas, les électeurs évaluent les performances économiques de leur point de vue personnel et dans le second du point de vue de l'ensemble de la société. Ce dernier comportement est qualifié de "sociotropique" et opposé à celui "d'égotropique".

Les très nombreuses études empiriques menées depuis le début des années 1970 n'ont pas permis de trancher entre les différents types de comportement ${ }^{5}$. Une partie de l'explication réside sans doute dans l'hétérogénéité de l'électorat, les électeurs réagissant différemment à l'évolution de la situation économique. Cette hétérogénéité peut prendre sa source dans l'asymétrie des montants d'information que possèdent les individus et la capacité qu'ils ont à la traiter. On peut aussi penser que l'absence de conclusion nette est liée à l'instabilité des comportements. En effet, un comportement particulier peut être mis en évidence sur certaines élections seulement et/ou dépendre du type d'élection.

\footnotetext{
${ }^{4}$ Les électeurs peuvent aussi utiliser les raccourcis cognitifs que constituent les partis politiques, les groupes de pression ou les médias (Popkin [1991]).

${ }^{5}$ Il est à noter que de très nombreuses autres dimensions du vote économique ont été étudiées parmi lesquelles l'asymétrie des jugements, le vote partisan (avec ses clivages vote "incubemcy oriented" / "policy oriented" et "hypothèse d'objectif prioritaire" / "hypothèse clientéliste"), l'altération du vote rétrospectif (clarté des responsabilités en présence de gouvernement de coalition ou de gouvernement éclaté et clarté de l'opposition), composante affective du vote, sophistication des électeurs, etc. On renvoie à Dubois [2005, chapitre 2] pour une revue très complète de la littérature.
} 
Ces grandes théories du comportement électoral ont été testées sur données micro dans le cas Français (voir entre autres, Lewis-Beck [1983, 1986a, 1988, 1996, 1997a] et LewisBeck et Nadeau [2000] ${ }^{6}$ ). La plupart de ces contributions mobilise une méthode similaire : pour chaque individu, le vote ou l'intention de vote est mis en relation avec divers indicateurs de la perception de l'évolution de la situation économique contrôlés par d'autres facteurs notamment de nature socio-démographique (proximité partisane, religion, CSP...). Les variables économiques, dites "subjectives", sont construites à partir de sondages d'opinion où l'on pose aux personnes interrogées les quatre questions suivantes définissant autant de comportement :

"Diriez-vous que depuis un an, votre situation financière s'est s'améliorée ?" (rétrospectifégotropique), "Pensez-vous que dans les douze mois qui viennent votre situation financière va s'améliorer ?" (prospectif-égotropique), "Diriez-vous que depuis un an, la situation économique du pays s'est améliorée ?" (rétrospectif-sociotropique), "Pensez-vous que dans les douze mois qui viennent la situation économique du pays va s'améliorer ?" (prospectifsociotropique). De manière générale, les corrélations entre le vote et les différents types de comportement peuvent être classées de la manière suivante : prospectif-sociotropique > rétrospectif-sociotropique > prospectif-égotropique > rétrospectif-égotropique. Il ressort donc que le vote égotropique est rejeté au profit du vote sociotropique. Pour la dimension temporelle, c'est moins net. Le vote prospectif semble dominer le vote rétrospectif mais cette supériorité n'est pas systématique.

Les études réalisées au niveau macro font l'objet de la suite du présent article. La première équation de vote française modélisant le vote agrégé est élaborée par Jean-Jacques Rosa et Daniel Amson et voit le jour en 1976, soit cinq ans après l'équation pionnière de Gerald Kramer. Avec cette étude, la France devient le deuxième pays à avoir son équation de vote et sera, avec les États-Unis et la Nouvelle-Zélande, l'un des trois pays étudiés au cours de la décennie 70 .

Comme chez Kramer [1971], l'équation de vote proposée par Rosa et Amson [1976] est l'œuvre d'économistes ${ }^{7}$ et elle a été publiée dans une revue de science politique. Ces équations partagent en outre la même structure, celle de Rosa et Amson [1976] étant une transposition au cas Français de celle de Kramer [1971].

\footnotetext{
${ }^{6}$ Pour une synthèse de ces études, on se reportera à Lewis-Beck et Stegmaier [2006].

${ }^{7}$ Du moins pour partie car Jean-Jacques Rosa est professeur d'économie à la FNSP et Daniel Amson est quant à lui juriste.
} 
A la suite de Rosa et Amson [1976], 71 contributions ayant pour objet les équations de vote ont, à notre connaissance, vu le jour pour le cas français. On peut répartir ces études de la manière suivante : 46 publiées dans des revues ou ouvrages académiques, 17 dans des journaux ${ }^{8}$ et 8 non publiées. Ce compte est approximatif car il y a des études non publiées auxquelles nous n'avons pas eu accès et donc qui ne sont pas comptées. A noter que parmi les études non publiées figurent deux thèses de doctorat (Auberger [2001] et Dubois [2005]) et que parmi les études publiées dans des revues ou ouvrages académiques figurent six surveys (Lewis-Beck et Stegmaier [2000], Lewis-Beck [2000, 2001, 2005], Bélanger [2004], Auberger [2004]) et trois réimpressions (Rosa [1980] pour Rosa et Amson [1976], LewisBeck et Rice [1992] pour Lewis-Beck [1991] et Lafay et Servais [2002] pour Lafay et Servais $\left.[2000]^{9}\right)$.

La première médiatisation d'un modèle dans un grand quotidien date de 1993 et d'un article consacré aux prévisions issues du modèle de Jérôme et al. [1993] ${ }^{10}$. Sur le plan académique, on peut noter qu'il a fallu près de vingt ans pour voir la publication d'un modèle de vote dans une revue d'économie française de premier plan (Auberger et Dubois [2003]).

Enfin, selon un dernier fait bibliométrique, on peut noter une nette accélération de la production scientifique sur les équations de vote (hors articles de journaux) : 1 étude dans les années 1970, 7 dans les années 1980, 15 dans les années 1990 et 31 depuis l'an 2000. Le nombre de contributions empiriques sur le vote a plus que doubler en moins de dix ans.

Le présent article se propose de faire un bilan de cette abondante littérature. Cet objectif a plusieurs fondements. Le premier, et le plus immédiat, est qu'il n'existe pas une telle revue de la littérature. Les contributions pré-citées, pour aussi synthétiques et pédagogiques soientelles, apparaissent partielles. Par exemple, la plus récente d'entre elles, Lewis-Beck [2005], ne comporte qu'une dizaine de références et ne contient aucune dimension historique (les études pionnières ne sont pas mentionnées). Ces contributions ont surtout pour objet une mise en perspective des expériences américaine et française de modélisation du vote. Ensuite, 2006 est l'année du trentième anniversaire de la première équation de vote estimée pour le cas Français. Enfin, 2007 va voir se succéder plusieurs échéances électorales qui vont être autant d'occasions de mettre à l'épreuve les modèles existants. Dans cette optique, il parait donc utile de réunir quelques éléments d'appréciation.

\footnotetext{
${ }^{8}$ Les articles de journaux ne sont pas cités en bibliographie mais le nom du journal et la date de parution sont indiqués dans le corps du texte ou en note de bas de page.

${ }^{9}$ Rosa [1980] est la version anglaise de Rosa et Amson [1976], Lewis-Beck et Rice [1992] incluent l'article de Lewis-Beck [1991] dans un ouvrage et Lafay et Servais [2002] est un reprint à l'identique de Lafay et Servais [2000] mais sous un titre différent.

${ }^{10}$ Le Figaro, 19/03/1993.
} 
L'article est divisé en cinq sections. Tout d'abord, le type et le niveau d'agrégation des élections étudiées sont examinés (section 1) puis les variables utilisées sont détaillées (section 2 pour la variable dépendante, section 3 pour les variables explicatives). Les problèmes économétriques rencontrés lors des estimations sont ensuite exposés (section 4). Enfin, les prévisions issues des équations de vote sont présentées (section 5).

\section{Les élections étudiées : type et niveau d'agrégation}

Entre 1976 et 1991, les études se sont focalisées exclusivement sur les élections nationales de premier plan : les élections législatives et les élections présidentielles. Peu à peu, les résultats des élections de tous types vont être modélisés. Le tableau 1 ci-dessous donne, pour chaque type, la première étude ayant vu le jour ainsi que le nombre total de contributions qui y sont consacrées $^{11}$.

\section{TABLEAU 1}

Premier modèle et nombre total de contributions pour chaque type d'élections

\begin{tabular}{lcc}
\hline \multicolumn{1}{c}{ Type } & Premier modèle & Nombre total de contributions \\
\hline Législatives & Rosa et Amson [1976] & 23 \\
Présidentielles & Lewis-Beck [1988] & 17 \\
Municipales & Lafay et Jérôme [1991] & 5 \\
Cantonales & Jérôme et Lewis-Beck [1999] & 2 \\
Régionales & Jérôme et Jérôme-Speziari [2000] & 3 \\
Européennes & Jérôme-Speziari et Jérôme [2003] & 4 \\
\hline
\end{tabular}

Les élections législatives et présidentielles, qui sont les plus importantes aux yeux des Français, restent les élections les plus étudiées.

Intéressons-nous maintenant au niveau d'agrégation des données utilisées. En France, le choix a longtemps été celui de données nationales et donc de techniques d'estimation de séries temporelles. En effet, le premier modèle en coupe instantanée a été publié en 1991 (Lafay et Jérôme [1991]) et le premier modèle utilisant des données de panel en 1993 (Jérôme et al. [1993]). Cet état de fait tranche avec l'expérience américaine pour laquelle la dernière classe de modèles a été utilisée dès le milieu des années 1970 (Meltzer et Vellrath [1975]). Depuis le

\footnotetext{
${ }^{11}$ Dans ce compte figurent uniquement les contributions présentant un modèle original (donc pas les reprints, ni les surveys) et les articles de journaux ne sont pas comptés. Dans le cas où une équation mélange plusieurs types d'élections, la contribution associée apparât une fois pour chaque type d'élections concerné. A noter que la première trace d'un modèle expliquant les résultats des élections européennes peut être trouvée en 1999 mais dans un article de journal (Le Figaro, 04/06/1999). De même, les premières prévisions présidentielles sont celles publiées dans Le Figaro / Études politiques de 1988 à partir du modèle législatif de Lewis-Beck [1985]. Enfin, précisons qu'il existe également un modèle explicatif des résultats de référendums (Jérôme et Vaillant [2005]).
} 
début des années 1990, on note une nette tendance à un plus grand recours à des modèles en données de panel ce qui, là encore, nous démarque des États-Unis où les équations de vote actuellement utilisées sont, dans leur très grande majorité, estimées sur données nationales.

L'utilisation de données nationales a un inconvénient majeur : le faible nombre d'observations et donc de degrés de liberté. Depuis le début de la cinquième République, il n'y a eu par exemple que sept élections présidentielles et douze élections législatives. "Pour l'économètre, on ne vote pas assez souvent" (Lafay [1995b], page 3). Ce problème a été rencontré bien vite par les premiers auteurs à s'être penchés sur les équations de vote en France et les a obligés à étendre leur période d'étude au-delà du début de la cinquième République (Rosa et Amson [1976], Lecaillon [1980], Lewis-Beck et Bellucci [1982] pour les élections législatives). Le manque d'homogénéité politique sur les périodes étudiées (changement de République, droit de vote des femmes...) affaiblissait considérablement la portée de ces équations obligeant même certains auteurs à corriger leur variable de vote (Lecaillon [1980]) ou à étendre leur échantillon à des élections d'un type semblable (élections constituantes ajoutées aux élections législatives chez Rosa et Amson [1976] et Lecaillon [1980] et référendum ajouté aux élections présidentielles chez Lewis-Beck [1988]). D'autres auteurs ont choisi, tout en conservant des données nationales, de mélanger plusieurs types d'élections. Ainsi, Lewis-Beck [1991] et Jérôme et Lewis-Beck [1999] proposent un modèle expliquant à la fois le résultat des élections législatives et présidentielles et Bélanger et LewisBeck [2004] et Lewis-Beck et Nadeau [2004] un modèle mélangeant élections législatives, présidentielles et européennes. Tous ces modèles reposent cependant sur l'hypothèse forte que les déterminants du vote sont les mêmes pour tous les types d'élections étudiés.

Pour palier le faible nombre de degrés de liberté, la grande majorité des chercheurs se sont tournés vers des modèles en coupe instantanée et en données de panel.

A notre connaissance, il existe sept modèles utilisant des données en coupe instantanée : Jérôme et Lafay [1991], Deffains et al. [1996], Palda et Palda [1998], Lafay et Servais [2000], Egal et al. [2003], Bonnetain [2004], Jérôme et Vaillant [2005] et Foucault et François [2005]. Cette méthode, qui nie le caractère temporel des évolutions, est mobilisée pour étudier l'impact de facteurs spécifiques comme par exemple de la qualité de la gestion lors des élections municipales de 1989 chez Jérôme et Lafay [1991], les taux d'imposition lors des élections municipales de 1995 chez Deffains et al. [1996], les scandales lors des élections municipales de 1995 chez Lafay et Servais [2000] ou encore les dépenses de campagne lors des élections législatives de 1997 chez Palda et Palda [1998] et Foucault et François [2005]. 
Les modèles en données de panel ont reçu une bien plus grande attention car, d'une part, ils permettent d'expliquer le vote d'une élection nationale ou locale à un niveau local, ce qui augmente le nombre de degrés de liberté, et d'autre part, car ils permettent de modéliser simultanément évolutions temporelles (d'une élection à l'autre) et disparités spatiales (entre territoires électoraux).

Le tableau 2 montre l'existence ou non d'un modèle pour expliquer chaque type d'élection ${ }^{12}$.

\section{TABLEAU 2}

Type de données utilisées pour expliquer chaque type d'élections

\begin{tabular}{lcccccc}
\hline \multicolumn{1}{c}{ Type } & \multicolumn{5}{c}{ Niveau des données } \\
\cline { 2 - 7 } \multicolumn{1}{c}{ d'ections } & Nation & Région & Département & Circonscription & Canton & Commune \\
\hline Municipales & non & non & non & non & non & oui \\
Cantonales & oui & oui & non & non & non & non \\
Régionales & non & oui & non & non & non & non \\
Législatives & oui & oui & oui & oui & non & non \\
Présidentielles & oui & oui & oui & non & non & oui \\
Européennes & oui & non & oui & non & non & non \\
\hline
\end{tabular}

Historiquement, les données désagrégées ${ }^{13}$ privilégiées ont été les données régionales (Jérôme et al. [1993, 1999, 2003], Jérôme et Jérôme-Speziari [2000, 2004], Fauvelle-Aymar et al. [2000]). Plus récemment, des modèles utilisant des données départementales ont vu le jour (Dubois [2002], Auberger et Dubois [2003, 2005], Bonnetain [2004], Auberger [2005]). Du point de vue théorique, les départements sont plus homogènes électoralement parlant que les régions en raison de leur découpage hérité de la Révolution de 1789 et qui respectait le découpage historique des diocèses. Les régions ne sont, quant à elles, apparues que bien plus tard et leur création correspondait plus à des problèmes d'aménagement du territoire. Le possible supplément d'homogénéité obtenu en descendant au niveau départemental est défendu par des politologues comme Bon et Cheylan [1988]. Cependant, du point de vue empirique, il semble difficile de mettre en évidence une homogénéité plus grande à l'intérieur des départements qu'à l'intérieur des régions. Par exemple, lorsqu'on examine les résultats du second tour des élections présidentielles de 1981 en métropole, il ressort (1) que 75 départements sur 96 ont voté dans le même sens que leur région de rattachement (soit 78,1\%)

\footnotetext{
${ }^{12}$ A noter que dans ce tableau n'apparaît pas la contribution d'Egal et al. [2003] qui est la seule à utiliser un niveau de données infra-communal puisqu'il s'agit d'un échantillon d'environ 500 quartiers (les IRIS2000 définis par l'INSEE).

${ }^{13}$ Dans ce qui suit, la terminologie "agrégé" (respectivement, "désagrégé") renvoie aux modèles utilisant des données au niveau national (respectivement, subnational).
} 
et (2) que 373 circonscriptions sur 555 ont voté dans le même sens que leur département de rattachement (soit 78,7 \%). Quoiqu'il en soit, en estimant un modèle identique (même variable expliquée, mêmes déterminants, même période) utilisant des niveaux de données différents (national, régional, départemental), Dubois et Fauvelle-Aymar [2004] ont montré une nette supériorité du modèle départemental en termes de prévision. Par ailleurs, le choix d'un niveau départemental garantit un plus grand nombre de degrés de liberté même s'il faut être vigilant à ce que cela ne conduise pas à l'abus de variables indicatrices.

\section{Le choix de la variable de vote}

Les premières équations de vote ont vu le jour aux États-Unis et se fondent donc sur un système quasi-bipartisan se réduisant à une lutte électorale entre le parti Démocrate et le parti Républicain. Les chercheurs ne retiennent généralement que le "two-party vote", c'est-à-dire le nombre de voix reçu par un parti exprimé en pourcentage du total des voix reçu par les deux principaux partis.

En France, un tel clivage entre deux grands partis n'existe pas et, consécutivement, on pourrait penser que les modèles américains du vote ne sont pas applicables au cas français. Cependant, une séparation héritée du XVIII ${ }^{\text {ème }}$ siècle demeure, celle qui divise le monde politique en deux sensibilités : gauche et droite. A quelques exceptions près ${ }^{14}$, on peut classer chaque parti politique sur une échelle gauche-droite et, selon les sondages de la SOFRES, environ $90 \%$ des sondés sont de nos jours capables de se positionner sur cette même échelle. Le clivage gauche-droite apparaît donc suffisamment marqué pour être retenu dans la construction des variables de vote à expliquer. Notons cependant que l'absence de clivage gauche-droite condamnerait l'entreprise de modélisation. C'est ainsi que l'absence de la gauche au second tour des élections présidentielles de 1969 et surtout de 2002 a conduit à la quasi-disparition des modèles explicatifs du vote présidentiel.

Il reste maintenant à déterminer qui de la gauche ou de la droite faut-il privilégier pour expliquer les résultats électoraux. En parcourant la littérature, on trouve aussi bien des modèles expliquant le vote en faveur des partis de gauche (Rosa et Amson [1976], Lecaillon [1980], Lewis-Beck et Bellucci [1982], Lewis-Beck [1985], Fauvelle-Aymar et al. [2000] ou Dubois et Fauvelle-Aymar [2004]) comme en faveur des partis de droite (Jérôme et al. [1993]). Selon une autre dimension, il existe des modèles expliquant le vote en faveur de

\footnotetext{
${ }^{14}$ On peut citer le cas des écologistes dans les années 1970 et les années 1980 et, plus récemment, celui du parti Chasse Pêche Nature Traditions.
} 
l'opposition (opposition au parti du Président pour les élections législatives chez Lewis-Beck $[1991,1995]^{15}$ ) ou du sortant. Dans ce dernier cas, il s'agit de la majorité sortante pour les élections législatives (Palda et Palda [1998], Lafay et Servais [2000], Jérôme et JérômeSpeziari [2000, 2002, 2004], Auberger [2001], Jérôme et al. [1999, 2001, 2003], Auberger et Dubois [2003, 2005], Bélanger et Lewis-Beck [2004], Lewis-Beck et Nadeau [2004], Dubois [2001, 2005], Foucault et François [2005]), du candidat du parti du Président sortant pour les élections présidentielles (Lewis-Beck [1988, 1991, 1995, 1997b], Courbis [1995], Auberger [2001], Dubois [2001, 2002], Jérôme et al. [2003], Bélanger et Lewis-Beck [2004], LewisBeck et Nadeau [2004] et Jérôme et Jérôme-Speziari [2004]), voire, pour ces mêmes élections, du candidat du parti du Premier ministre sortant (Courbis [1995], Auberger [2001]). La tendance à expliquer le vote pour le sortant est donc récente. Elle n'en n'est pas moins dominante de nos jours. Ceci provient sans doute du fait que la théorie sous-jacente de tous ces modèles est le vote rétrospectif. Or, comme nous l'avons vu précédemment, le vote rétrospectif concerne le sortant. Il est donc logique que la variable dépendante soit le vote en faveur du sortant lorsque le fondement théorique privilégié est le vote rétrospectif. Le fait que certains modèles aient choisi une autre option, comme modéliser le vote en faveur de la gauche par exemple, tient au fait que, longtemps, le vote à gauche a été assimilé à un vote de protestation. On peut enfin remarquer que la très grande majorité des modèles étrangers ont eux aussi opté pour une variable de vote en termes de sortant.

La dernière question ayant trait au choix de la sensibilité pertinente concerne le statut de l'extrême droite. Jusqu'au début des années 1990, les modèles ne la traitaient pas de manière particulière car elle ne représentait que quelques points de vote. Même lorsque ses scores ont commencé à s'élever, aucune modélisation spécifique n'a été proposée puisque par le jeu du scrutin majoritaire, l'extrême droite n'arrivait pas à convertir ses voix du premier tour en sièges à l'issue du second. Les élections législatives de 1986 mises à part ${ }^{16}$, l'extrême droite n'a obtenu en tout et pour tout que deux sièges à l'Assemblée Nationale depuis le début de la cinquième République $^{17}$. Malgré cela, l'extrême droite joue néanmoins un rôle perturbateur en gênant souvent la droite modérée. C'est notamment le cas dans les fameuses triangulaires qui voient s'affronter un candidat de gauche, un candidat de droite modérée et un candidat d'extrême droite. Les électeurs d'extrême droite portent leur voix sur le candidat de l'extrême

\footnotetext{
${ }^{15}$ Pour ces deux dernières contributions, il s'agit du même modèle. Celui-ci faisait suite à celui de Lewis-Beck [1985] sans qu'il n'y ait de contradiction puisque avant 1981, expliquer les résultats en faveur de la gauche et expliquer les résultats en faveur de l'opposition revenait au même.

${ }^{16}$ Lors de ce scrutin à la proportionnelle, l'extrême droite a obtenu 35 sièges.

17 siège en 1988 et 1 siège en 1997.
} 
droite alors que dans le cas d'un duel classique gauche / droite modérée, ils auraient voté pour le candidat de la droite modérée. Dans le cas de triangulaire, la droite modérée se retrouve donc fréquemment minoritaire par rapport à la gauche. La plupart des modèles législatifs ont fait le choix d'expliquer les résultats de la droite entière qu'elle soit la majorité sortante ou pas (Jérôme et al. [1993, 1999, 2003], Auberger et Dubois [2003, 2005], Jérôme et JérômeSpeziari [2004]). Les seules exceptions connues sont Dubois [2001, 2005] mais l'auteur explique les résultats au second tour, tour auquel l'extrême droite fait traditionnellement des scores négligeables. On peut noter également la modélisation particulière d'Auberger [2001], Bélanger et Lewis-Beck [2004] et Lewis-Beck et Nadeau [2004] qui retiennent une mesure des votes proche du "two-party vote" américain : la part de la gauche ou de la droite modérée est exprimée en pourcentage de la somme des voix de la gauche et de la droite modérée. Pour les modèles retenant le vote de la droite entière, il apparaît donc nécessaire de corriger les voix obtenues. Dans le cadre des modèles législatifs, cette correction passe par la prise en compte de l'extrême droite dans les équations de sièges sur lesquelles nous reviendrons ultérieurement. Une autre possibilité est de construire une équation de vote expliquant le vote en faveur de l'extrême droite. Par simple soustraction, il est alors facile d'obtenir le vote en faveur de la droite modérée. Une telle équation de vote pour l'extrême droite a été estimée par Auberger [2005] pour les élections européennes, Jérôme et Jérôme-Speziari [2003, 2004] et Bonnetain [2004] pour le premier tour des élections présidentielles, Fauvelle-Aymar et LewisBeck [2005] pour le premier tour des élections législatives et Jérôme et Jérôme-Speziari [2003] pour les élections régionales ${ }^{18}$. Pour finir avec l'extrême droite, notons qu'elle pose un sérieux problème aux modèles expliquant le vote en faveur de la gauche ou le vote en faveur de l'opposition, notamment si ceux-ci sont utilisés à des fins de prévision. En effet, prenons le cas du célèbre modèle de l'Iowa ${ }^{19}$ qui explique et prévoit les résultats en faveur des partis opposés au Président. Par le jeu de l'extrême droite, ce modèle ne peut pas dire qui de la gauche ou de la droite sera au pouvoir. Par exemple, prévoir $53 \%$ pour l'opposition si la gauche est au pouvoir peut signifier aussi bien une victoire de celle-ci qu'une défaite (selon que l'extrême droite obtient plus ou moins de $7 \%$ des voix).

Enfin, pour les scrutins à deux tours, il convient de s'interroger sur le tour à retenir pour expliquer les résultats électoraux. C. de Gaulle justifiait le scrutin à deux tours en disant que

\footnotetext{
${ }^{18}$ Notons qu'en dehors de ces fonctions de vote extrême droite, il n'existe, à notre connaissance, que trois contributions ayant tenté d'expliquer le vote en faveur d'un parti particulier, le modèle présenté dans l'article du Figaro du 04/06/1999 et Jérôme-Speziari [2003] qui étudient le vote socialiste et celle de Égal et al. [2003] qui étudie le vote écologiste.

${ }^{19}$ Voir Libération du 23/05/1997. Pour une discussion autour de ce modèle, voir Cayrol et al. [1997].
} 
cela permettait aux gens de "voter avec leur cœur dans un premier temps et de voter avec leur portefeuille ensuite" (cité par Norpoth [1996], page 312). Les résultats du premier tour reflèteraient donc mieux les préférences réelles des électeurs et le second tour les perturberait par la réduction de l'offre politique et les stratégies d'entre deux tours qu'il entraîne. Cependant, lorsqu'on examine les résultats des élections législatives par exemple, on s'aperçoit qu'arriver en tête au premier tour ne garantit pas la majorité à l'issue du second et que c'est bien ce dernier qui "fait l'élection". Pour ne citer que deux exemples, la gauche arrive en tête au soir du premier tour des élections de 1978 mais est finalement battue au second. Inversement, en 1988, elle rate de peu la barre des $50 \%$ de voix au premier tour mais se retrouve largement majoritaire à l'Assemblée Nationale. Cet état de fait est très probablement dû d'une part au rôle perturbateur joué par l'extrême droite et d'autre part, au très grand nombre de circonscriptions concernées par un second tour (le pourcentage moyen de circonscriptions en ballottage pour les 12 élections législatives de la cinquième République est de $85 \%^{20}$ ).

Quoi qu'il en soit, on trouve un peu toutes les modélisations dans la littérature : premier tour des présidentielles chez Fauvelle-Aymar et al. [2000], Jérôme et al. [2003] et Jérôme et Jérôme-Speziari [2004], second tour des présidentielles chez Lewis-Beck [1988, 1991, 1995, 1997b], Courbis [1995], Auberger [2001], Dubois [2001, 2002], Bélanger et Lewis-Beck [2004], Lewis-Beck et Nadeau [2004], Jérôme et Jérôme-Speziari [2004], premier tour des législatives chez Rosa et Amson [1976], Lecaillon [1980], Lewis-Beck et Bellucci [1982], Lewis-Beck [1985, 1991,1995], Jérôme et al. [1993], Palda et Palda [1998], Jérôme et al. [1999, 2001, 2004], Fauvelle-Aymar et al. [2000], Auberger [2001], Bélanger et Lewis-Beck [2004], Lewis-Beck et Nadeau [2004], Jérôme et Jérôme-Speziari [2004] et Foucault et François [2005], second tour des législatives chez Dubois [2001, 2005]. On trouve également des études qui retiennent soit un tour, soit l'autre selon si un tour ou deux a été nécessaire (cas des études municipales de Jérôme et Lafay [1991] et Jérôme et Jérôme-Speziari [2002]). Enfin, Auberger et Dubois [2003, 2005] agrègent pour leur modèle législatif départemental le vote reçu au premier tour dans les circonscriptions où un seul tour a été nécessaire et le vote reçu au second tour dans les circonscriptions où deux tours ont été nécessaires.

\section{Les déterminants du vote}

\footnotetext{
${ }^{20}$ Sources : Lancelot [1998] de 1958 à 1997, www.robert-schuman.org pour 2002. A noter : un seul tour pour 1986.
} 


\subsection{Les variables économiques}

Dans la lignée de Kramer [1971] pour les États-Unis, les premiers modèles français comportent uniquement des variables économiques (Rosa et Amson [1976], Lecaillon [1980], Lewis-Beck et Bellucci [1982]). Ces travaux utilisent les grandes variables macroéconomiques (chômage, prix et revenu) pouvant être définies en niveau ou en variation et sur une ou plusieurs années. A partir du milieu des années 1980, ces variables sont abandonnées notamment à cause de la multicolinéarité que leur introduction simultanée pouvait entraîner ${ }^{21}$. Par ailleurs, les variables de prix, dont le statut apparaissait flou (l'inflation signifie une hausse des prix mais elle est aussi associée aux périodes d'expansion économique), ont aujourd'hui disparu.

Le choix s'est porté sur la croissance du PIB, une variable que les anglo-saxons qualifient de "valence issue", c'est-à-dire une variable sur laquelle tous les agents ont la même opinion quant à son évolution (tous les agents veulent plus de croissance).

A la suite de Lewis-Beck [1985], la très grande majorité des modèles nationaux ayant pour ambition d'expliquer le vote lors des élections législatives ou présidentielles emploieront cette variable (Lewis-Beck [1991, 1995, 1997b], Courbis [1995], Auberger [2001], Jérôme et al. [2001], Dubois [2005]). Cependant, l'emploi des données issues de la Comptabilité Nationale et en particulier des données trimestrielles soulève deux problèmes: celui des changements de base récurrents qui posent la question de la base pertinente à retenir et celui de l'utilisation de données trimestrielles pour expliquer le vote depuis 1958 alors que les comptes nationaux trimestriels n'ont été élaborés qu'à partir du milieu des années 1970.

Pour les modèles utilisant des données désagrégées, c'est un peu différent car les données de PIB au niveau régional ou départemental ne sont pas disponibles sur une période suffisamment longue pour être utilisées. Par ailleurs, elles n'ont longtemps été connues qu'avec retard, ce qui pouvait entraîner des problèmes si le modèle était utilisé à des fins de prévision. Ces deux difficultés expliquent sans doute qu'aucun modèle existant n'utilise les séries du PIB local. A notre connaissance, le PIB apparaît dans deux modèles utilisant des données de panel (Auberger et Dubois [2003, 2005] et Auberger [2005]) et dans les deux cas, il s'agit de la variation de la croissance au niveau national qui sert à expliquer le vote au niveau départemental. En dehors du PIB, on trouve une autre variable de Comptabilité Nationale : le revenu disponible brut des ménages (Auberger [2001], Dubois [2001]).

\footnotetext{
${ }^{21}$ Notons que l'on trouve encore parfois la variable de chômage seule comme chez Jérôme et al. [2001].
} 
La variable économique privilégiée par les modèles désagrégés est le chômage (Jérôme et al. [1993, 1999, 2003], Jérôme et Jérôme-Speziari [2000, 2002, 2004], Dubois [2002], Dubois et Fauvelle-Aymar [2004], Auberger et Dubois [2003, 2005], Auberger [2005]). Dans tous ces modèles, il s'agit de la variation du taux de chômage excepté chez Jérôme et JérômeSpeziari [2000] où il s'agit de l'écart entre le taux de chômage régional et le taux de chômage national et chez Dubois [2002], Auberger et Dubois [2003, 2005] et Auberger [2005] où il s'agit du taux de variation du nombre de demandeurs d'emploi par département.

En dehors de ces grandes variables macroéconomiques, on trouve des variables fiscales surtout utilisées dans des modèles expliquant le vote à un niveau municipal (Deffains et al. [1996], Lafay et Servais [2000], Jérôme et Jérôme-Speziari [2002]) ou départemental (Bonnetain [2004]). Notons également l'approche originale de Jérôme et al. [2001] qui consiste à expliquer le vote lors des élections législatives françaises par des variables économiques définies comme la différence entre la valeur de la variable en France et en Allemagne.

Toutes les variables économiques que nous avons mentionnées jusqu'à présent sont des variables objectives ; il s'agit de mesures effectives de l'état de l'économie. Il existe également, comme nous l'avons vu, des variables subjectives construites à partir de réponses à des sondages d'opinion. On demande aux sondés de donner leur avis sur la situation passée ou future de leur ménage ou de l'économie dans son ensemble. Les pourcentages obtenus permettent de définir des variables surtout utilisées dans les études sur données individuelles. Il n'existe, à notre connaissance, que deux études les ayant introduits dans une étude sur données agrégées (Bélanger et Lewis-Beck [2004] et Dubois [2005]). Si toutes deux ont conclu que les variables économiques subjectives étaient bien pertinentes pour expliquer le vote, Dubois [2005] a cependant montré que, contrôlées par la popularité politique ${ }^{22}$, ces variables ne dominent pas, en termes de pouvoir explicatif, les variables économiques objectives.

Sur le plan théorique, on constate que les variables économiques sont presque exclusivement des variables objectives (sauf bien entendu lorsqu'il s'agit d'étudier l'impact des variables subjectives). Par ailleurs, ces variables sont toutes à caractère sociotropique dans le sens où elles concernent un ensemble d'individus. On remarque cependant que, pour expliquer le vote au niveau local, le chômage local donne de meilleurs résultats que le

\footnotetext{
${ }^{22}$ On peut s'interroger sur d'éventuels problèmes de spécifications dans l'étude de Bélanger et Lewis-Beck [2004] car une variable incontournable comme la popularité est omise. Par ailleurs, l'échantillon présente un biais de sélection, les élections européennes de 1979 n'étant pas incluses (pour une raison indéterminée).
} 
chômage national. Les électeurs semblent ainsi plus préoccupés par la situation économique qui les touche plus directement que par la situation économique nationale. On peut donc parler d'un vote sociotropique atténué. Enfin, concernant la dimension temporelle, les modèles valident dans leur très grande majorité le vote rétrospectif. Des exceptions peuvent cependant être trouvées comme par exemple chez Lewis-Beck [1988, 1991, 1997] et Auberger et Dubois [2003, 2005] où la définition de certaines variables économiques englobe l'année des élections. Au moment du scrutin, ces données ne sont pas connues et nécessitent une anticipation qui donne au vote un caractère prospectif.

Puisque les variables utilisées sont généralement rétrospectives, il est intéressant de regarder sur combien de temps porte leur définition. Ceci traduit en effet le degré de mémoire des électeurs. A notre connaissance, il n'existe pas de véritable contribution théorique sur le sujet et la littérature est en conséquence exclusivement empirique. Les auteurs estiment des fonctions de vote en envisageant différents retards pour leurs variables et sélectionnent les retards offrant le plus grand pouvoir explicatif. Si l'on examine la définition de la variable économique utilisée dans les 25 principales études sur le vote législatif ou présidentiel, on constate que l'horizon est relativement court : dans aucun modèle il n'atteint deux ans. Il varie en effet de un trimestre chez Fauvelle-Aymar et Dubois [2004] à sept trimestres chez Auberger [2001]. La moyenne est quant à elle légèrement supérieure à un $a^{23}$. Sur la foi de ces modèles, la mémoire de l'électeur Français apparaît donc limitée. Les études pour les autres pays, et notamment les États-Unis, aboutissent généralement à la même conclusion.

\subsection{Les variables institutionnelles}

Un des traits particuliers du système politique français est la possibilité de cohabitation, une situation dans laquelle le Premier ministre et le Président de la République n'appartiennent pas au même bord politique. Du point de vue théorique, cette division de l'exécutif brouillerait la capacité de jugement des électeurs qui ne seraient plus capables d'attribuer la responsabilité du bilan économique (Downs [1957], chapitre 9). Donc lorsque la responsabilité est diffuse, le vote économique serait atténué. Quatre contributions sur le vote au niveau agrégé ont étudié l'impact de la cohabitation et confirment la prescription théorique ci-dessus : Lewis-Beck

\footnotetext{
${ }^{23}$ Ces chiffres concernant la mémoire incluent la durée sur laquelle est définie la variable et son décalage par rapport aux élections.
} 
[1997b] dans le cas des élections présidentielles, Lewis-Beck et Nadeau [2004] ${ }^{24}$ et Bélanger et Lewis-Beck [2004] pour les élections européennes, présidentielles et législatives et Dubois [2005] pour les élections législatives.

Le second facteur institutionnel important est la modification de la règle électorale lors des élections législatives de 1986 qui se sont déroulées selon la règle de la proportionnelle à un tour. La seule étude à avoir pris en compte ce changement dans une fonction de vote, Dubois [2005], ne permet pas de conclure à un effet spécifique de cette modification sur le vote.

\subsection{Les variables politiques}

Les principales variables politiques sont au nombre de trois : l'abstention, le vote lors des élections précédentes et la popularité.

L'abstention (ou son complément la participation) est une variable explicative du vote présente notamment chez Fauvelle-Aymar et al. [2000] et Dubois [2005]. Dans la première étude, la participation a un impact positif sur le vote à gauche, suggérant ainsi que les abstentionnistes et les électeurs de gauche partagent des caractéristiques communes. Dubois [2005] détecte un autre effet : le vote pour la majorité sortante au second tour des élections législatives et l'abstention sont corrélés négativement. On peut penser que des électeurs mécontents de la majorité au pouvoir s'abstiennent plutôt que de voter contre surtout s'ils sont du même bord politique qu'elle. Inversement, on peut aussi penser qu'un très mauvais bilan de la majorité sortante peut amener aux urnes des personnes qui habituellement s'abstiennent.

Dans de nombreuses études figurent les élections précédentes dont les résultats peuvent retranscrire la force électorale tantôt de court terme, tantôt de long terme. En effet, d'un côté, pour les élections législatives par exemple, on observe que, dans la grande majorité des cas, les élections précédentes, quel que soit leur type (cantonales, présidentielles...), annoncent le sens de la majorité issue des élections législatives à venir (Dubois [2001, 2002], Auberger et Dubois [2003, 2005]). On trouve notamment le phénomène bien connu des élections législatives suivant immédiatement des élections présidentielles qui constituent des élections de confirmation $(1981,1988,2002)$. D'un autre côté, si l'on considère le résultat des élections précédentes de même type, c'est-à-dire un vote qui se reporte d'une élection à l'autre, on peut l'interpréter comme la transcription d'un vote à long terme, une sorte d'inertie dans le vote,

\footnotetext{
${ }^{24}$ Comme évoqué précédemment, on peut s'interroger sur d'éventuels problèmes de spécification car, mise à part la cohabitation, le vote ne dépend que de variables économiques.
} 
inertie pouvant être expliquée par la force du vote partisan : quelle que soit la conjoncture économique ou politique, il y a des électeurs qui votent toujours dans le même sens. Dans ce cas, le vote lors des élections précédentes n'est qu'une variable instrumentale, une proxy pour des facteurs socio-démographiques influençant le vote sur le long terme comme la catégorie socio-professionnelle ou la religion. Dans certains pays pour lesquels les données sont disponibles sur une période suffisamment longue, la proximité partisane est également utilisée. Il s'agit du pourcentage de personnes se déclarant proches d'un parti particulier. Cette variable est un substitut à celle des élections précédentes car toutes deux ont les mêmes déterminants socio-démographiques. On peut enfin remarquer que dans un modèle utilisant des données de panel, les effets fixes peuvent se substituer à la variable d'élections précédentes (Dubois et Fauvelle-Aymar [2004]).

La dernière grande variable politique est la popularité. A la suite de Lewis-Beck [1985], la plupart des études françaises la prennent en compte en complément d'une variable macroéconomique $^{25}$. La popularité possède l'avantage de capter l'influence de facteurs difficiles à appréhender quantitativement comme le style, le charisme ou la personnalité mais aussi la réaction des agents à des prises de position sur des questions de société. Cependant, son principal avantage est également son principal inconvénient. En effet, de par son caractère "fourre tout", elle englobe aussi le jugement des sondés sur les résultats économiques du gouvernement ce qui peut poser des problèmes de multicolinéarité lorsque elle est entrée dans un modèle simultanément avec une ou plusieurs variables économiques.

Il existe d'autres variables politiques qui n'apparaissent pas dans les études françaises et que l'on rencontre fréquemment à l'étranger comme le nombre de mandats effectués par le sortant (notamment aux États-Unis). Cette absence provient sans doute de la difficulté d'interprétation de ce type de variables qui traduisent aussi bien une prime au sortant qu'une usure du pouvoir. On peut aussi penser que la France n'offre aucune régularité en la matière : aucune majorité législative reconduite depuis 1978, certains Présidents de la République briguant un second mandat réélus (V. de Gaulle, F. Mitterrand, J. Chirac) et d'autres pas (V. Giscard-d'Estaing), etc.

\subsection{Le contexte de l'élection}

\footnotetext{
${ }^{25}$ La variable de popularité est également présente dans la plupart des modèles développés pour les autres pays (parfois remplacer par les intentions de vote). Par ailleurs, ce n'est pas le hasard si la structure des équations de vote composée de deux variables explicatives, une variable macroéconomique et une variable de popularité politique, s'est imposée en France : elle a été introduite dans notre pays par M. Lewis-Beck, l'un des deux chercheurs ayant popularisé cette approche aux États-Unis (Lewis-Beck et Rice [1984]).
} 
En dehors des variables économiques, institutionnelles et politiques, les études sur les déterminants du vote en France incluent plusieurs autres variables destinées à capter le contexte de l'élection. On en trouve principalement trois : les dépenses de campagne chez Palda et Palda [1998] et Foucault et François [2005], les scandales chez Lafay et Servais [2000] et le localisme chez Dubois [2002].

En France, les dépenses des partis politiques et des candidats ne sont publiées que depuis 1990, année de la création de la Commission Nationale des Comptes de Campagne. Comme il est impossible de réunir des données sur un nombre conséquent d'élections, les dépenses de campagnes ne sont généralement pas incluses dans les modèles. Leur impact sur le vote n'a été étudié que dans le cadre de modèle en coupe instantanée et uniquement pour les élections législatives de 1997 (Palda et Palda [1998] et Foucault et François [2005]).

L'absence de données homogènes sur une période suffisamment longue empêche également l'étude de l'influence des scandales sur le vote dans le cadre de modèles agrégés ou en données de panel. Lafay et Servais [2000] ont cependant montré l'impact négatif des scandales sur le vote lors des élections municipales de 1995 pour 92 villes.

Les caractéristiques des candidats font également partie du contexte de l'élection. Selon la théorie du localisme, les candidats auraient un avantage électoral dans les territoires dont ils sont issus. Ceci semble effectivement être le cas en France pour les élections présidentielles (Dubois [2002]).

Avant de clore ce paragraphe sur le contexte de l'élection, examinons le rôle de la campagne, sujet à de nombreuses controverses. Généralement, on considère que la campagne est spécifique à chaque élection car les candidats et surtout les enjeux sont différents d'une élection à l'autre. La campagne apparaît donc comme un facteur idiosyncrasique et ne peut donc être modélisée que par un terme aléatoire.

Selon un autre argument, hérité des travaux de Lazarsfeld et al. [1944] aux États-Unis, la campagne ne joue qu'à la marge car peu d'électeurs changent d'opinion au cours de celle-ci. Selon eux, ceci est la conséquence du poids très fort de l'identification partisane. Si les électeurs se décident majoritairement bien avant le jour des élections et en fait avant le début de la campagne électorale, alors il convient de retenir des variables économiques et politiques définies plusieurs mois avant les élections. En France, cette option a été choisie par, entre autres, Lewis-Beck [1985, 1988, 1991, 1995, 1997b]. Cependant, cela ne semble plus très adapté à la période récente car les électeurs français se décident de plus en plus tard quant à 
leur vote. Par exemple, lors des élections présidentielles de 2002, une semaine avant le premier tour, $32 \%$ des personnes interrogées déclaraient pouvoir changer d'avis ${ }^{26}$.

Enfin, pour d'autres auteurs, la campagne électorale est prise en compte de manière implicite au travers des variables économiques (Dubois [2005]). En effet, selon certaines études, la campagne électorale apporte aux électeurs des informations essentiellement sur la situation économique (voir, entre autres, Paldam et Nannestad [2000]).

Avant de clore cette section consacrée à la structure des équations de vote en France, il faut mentionner l'existence d'une modélisation complémentaire, l'équation de sièges. En effet, dans le système représentatif français - le système majoritaire - les voix seules ne permettent pas toujours de connaître le sens de la future majorité. Par exemple, avec 44,6 \% des voix, la droite modérée a remporté les élections législatives de 1986 mais a perdu celles de 1997 avec un pourcentage plus élevé (46,1\% au second tour). C'est pour cela qu'il est nécessaire de construire une fonction de sièges permettant de transformer les voix en sièges. La modélisation des sièges est souvent rudimentaire et, dans une même étude, le pouvoir explicatif de la fonction de sièges est quasi systématiquement moindre que celui de la fonction de vote. La plupart du temps, il s'agit simplement de lier par une relation linéaire voix et sièges (Lewis-Beck [1985, 1991], Jérôme et Lewis-Beck [1999], Jérôme et JérômeSpeziari [2000], Auberger [2001] et Dubois [2001]). Les fonctions de sièges vont s'affiner avec les modèles en données de panel. On voit apparaître trois facteurs principaux : la prise en compte du rôle perturbateur de l'extrême droite (Jérôme et al. [1993, 1999, 2003], Auberger et Dubois [2003, 2005]), des conséquences de la modification de la règle de vote en 1986 (Jérôme et al. [1993]) et de l'impact de l'abstention (Jérôme et al. [1999, 2003], Jérôme et Jérôme-Speziari [2004]).

\section{Les problèmes économétriques}

Si l'on se borne aux grands problèmes économétriques que sont l'auto-corrélation des résidus, leur normalité, l'hétéroscédasticité, les phénomènes $\mathrm{ARCH}$, la stationnarité ou la cointégration, force est de constater que leur traitement dans les études sur le vote, s'il existe, est relativement fruste. Par exemple, auto-corrélation ${ }^{27}$, phénomènes $\mathrm{ARCH}$ et stationnarité ne sont pris en compte que chez Dubois [2001, 2005] dans le cadre de modèles utilisant des

\footnotetext{
${ }^{26}$ Selon un sondage BVA réalisé du 10 au 13 avril 2002.

${ }^{27}$ On entend ici l'ensemble des corrélations (test de Ljung-Box par exemple) et pas seulement le premier retard (test de Durbin-Watson).
} 
séries temporelles. De même, l'hétéroscédasticité des résidus, problème majeur rencontré lors de l'utilisation de coupe instantanée, est négligé dans toutes les études mentionnées plus haut à l'exception de Foucault et François [2005]. Concernant les estimations sur données de panel, deux études ont vérifié l'absence d'auto-corrélation des résidus (Jérôme et al. [1999] et Auberger et Dubois [2005]) et une seule a pris en compte la possibilité de phénomènes ARCH (Jérôme et al. [1999]). Par ailleurs, à notre connaissance, aucun modèle utilisant des données de panel n'a fait l'objet d'une étude de stationnarité ou de cointégration. Enfin, le diagnostic de la normalité résidus, pourtant capital, n'a été effectué que chez Auberger et Dubois [2005] et chez Dubois [2005].

Toujours concernant l'économétrie, nous souhaiterions faire deux remarques. La première concerne le traitement qui est réservé à la constante dans les modèles en données de panel. L'avantage des données de panel est précisément de permettre une plus grande flexibilité dans la constante. Notamment, on distingue trois types de modèles où i désigne l'unité territoriale (région, département...) et t la date de l'élection :

$$
\begin{gathered}
\mathrm{Y}_{\mathrm{it}}=\alpha+\beta \mathrm{X}_{\mathrm{it}}+\varepsilon_{\mathrm{it}} \\
\mathrm{Y}_{\mathrm{it}}=\alpha_{\mathrm{i}}+\beta \mathrm{X}_{\mathrm{it}}+\varepsilon_{\mathrm{it}} \\
\mathrm{Y}_{\mathrm{it}}=\alpha+\mathrm{u}_{\mathrm{i}}+\beta \mathrm{X}_{\mathrm{it}}+\varepsilon_{\mathrm{it}}
\end{gathered}
$$

Le premier est un modèle "sans effet" : la constante est la même pour toutes les unités territoriales à toutes les dates. Le second modèle est dit "à effets fixes". Cette fois-ci, la constante est la même d'une date à l'autre mais elle varie d'une unité territoriale à l'autre. Ce modèle est équivalent à un modèle dans lequel on aurait inclus i variables indicatrices valant 1 pour une unité territoriale particulière et 0 ailleurs. Enfin, le dernier modèle est le modèle "à effets aléatoires". Il y a à la fois une constante identique pour toutes les unités territoriales et pour toutes les dates et un terme aléatoire spécifique à chaque unité territoriale. Très peu de fonctions de vote estimées sur données de panel intègrent cette dimension de flexibilité dans la constante et mettent en œuvre les tests adéquats ${ }^{28}$ (cinq sur les quatorze contributions concernées : Auberger et Dubois [2003, 2005], Dubois et Fauvelle-Aymar [2004], Auberger [2005] et Fauvelle-Aymar et Lewis-Beck [2005]).

La seconde remarque est le problème posé par l'estimation, dans une même contribution, d'une fonction de vote et d'une fonction de sièges. Ces deux équations forment en fait un système que l'on peut représenter schématiquement de la manière suivante :

\footnotetext{
${ }^{28}$ Test de Fisher pour trancher entre modèle sans effet et modèle à effets fixes, test du multiplicateur de Lagrange pour trancher entre modèle sans effet et modèle à effets aléatoires et test d'Hausman pour trancher entre modèle à effets fixes et modèle à effets aléatoires.
} 


$$
\begin{gathered}
\text { VOTE }=\mathrm{f}(\mathrm{W}) \\
\text { SIEGES }=\mathrm{g}(\text { VOTE }, \mathrm{Z})
\end{gathered}
$$

où $\mathrm{W}$ et $\mathrm{Z}$ sont des vecteurs de variables explicatives. Pour estimer un tel système d'équations, plusieurs méthodes peuvent être mobilisées comme le maximum de vraisemblance à information complète (FIML) ou les triples moindres carrés même si on peut noter que les MCO peuvent être utilisés sous hypothèse d'indépendance des résidus d'une équation à l'autre. A notre connaissance, une telle hypothèse n'a jamais été formulée dans une contribution présentant une équation de vote et une équation de sièges et seuls Auberger et Dubois [2005] ont employé une technique d'estimation de système pour estimer leurs deux équations (en l'occurrence, la méthode FIML) ${ }^{29}$.

\section{Les performances des modèles en terme de prévision}

Cette cinquième et dernière section est consacrée à une utilisation particulière des fonctions de vote : la prévision des résultats électoraux ${ }^{30}$. Cette utilisation est ancienne puisqu'elle remonte à Lecaillon [1980] qui proposait plusieurs scénarios pour les élections législatives prévues, à l'époque, en 1983.

Mais l'entreprise de prévision a réellement pris son essor avec Lewis-Beck [1985] qui annonçait la défaite de la gauche aux élections législatives de 1986 avec 46,9\% des voix en se basant sur les valeurs anticipées des variables de son modèle. Avec les vraies valeurs, la prévision était de 46,0 \% (Lewis-Beck [1986b], page 32). La gauche a finalement obtenu 45,3 $\%^{31}$. Les derniers sondages publiés avant le scrutin donnaient $44,5 \%$ pour la SOFRES, 46,0 pour BVA et $46,5 \%$ pour l'IFOP.

Les modèles nationaux connaîtront par la suite des fortunes diverses, notamment en ce qui concerne l'ampleur de l'erreur de prévision. On peut noter le succès du modèle présidentiel de Lewis-Beck [1988] qui prévoyait la réélection de François Mitterrand en 1988 avec entre 52 et $53 \%$ des voix au second tour alors qu'il a obtenu $54 \%$. A notre connaissance, aucune prévision n'a été diffusée pour les élections législatives de 1993 mais à partir des coefficients

\footnotetext{
29 A noter toutefois que Fauvelle-Aymar et al. [2000] appliquaient la technique des 2SLS pour estimer leur système à équations simultanées vote-abstention.

${ }^{30}$ Nous nous intéressons ici pour l'essentiel aux prévisions ex ante, c'est-à-dire calculées avant le scrutin (qu'elles aient été diffusées avant ou pas). Dans les études, d'autres indicateurs de performance sont présentés comme des simulations historiques, des prévisions ex post, des probabilités de réélection ou des intervalles de confiance (voir, entre autres, Dubois [2001, 2005]).

${ }^{31}$ Ici et dans ce qui suit, la valeur réalisée pour les voix est celle résultant de nos calculs à partir de Lancelot [1998].
} 
du modèle proposé par Lewis-Beck [1991], on pouvait calculer une prévision d'environ 55 \% pour l'opposition au premier tour qui a en fait obtenu $57 \%$. Les principaux instituts de sondages avaient prévu correctement l'issue du scrutin : 52,5\% pour l'IFOP, 53,5\% pour la SOFRES et 55,0 \% pour BVA. Pour les élections présidentielles de 1995, plusieurs prévisions issues d'équations de vote ont été publiées donnant le candidat de gauche ou le candidat de droite gagnant selon le modèle (Lewis-Beck [1995, 1997b]) ${ }^{32}$. Vient ensuite le cas singulier des élections législatives de 1997. Alors qu'avant le premier tour, tous les sondages d'intentions de vote donnaient la droite vainqueur ${ }^{33}$, le modèle de C. Fauvelle-Aymar et M. Lewis-Beck, baptisé "modèle de Iowa", prévoyait la victoire de l'opposition avec 56,9 \% au premier tour ${ }^{34}$. Si c'est sans nul doute un succès sur le plan du résultat final du scrutin, on constate une forte erreur de prévision (l'opposition a obtenu 62,6\% des voix) à laquelle s'ajoute une incertitude dont nous avons déjà parlé liée à la répartition des voix entre gauche et extrême droite. En 2002, un modèle donnait le candidat de gauche très légèrement vainqueur aux élections présidentielles ${ }^{35}$ et celui de Dubois [2001] prédisait alternativement la victoire du candidat de gauche et du candidat de droite pour ces mêmes élections dans les mois précédant le scrutin. Ce dernier modèle n'a pas fait mieux lors des élections législatives en commettant une erreur de près de trois points malgré un sens de la future majorité correctement prévu. Les modèles agrégés sont probablement morts.

Comme nous l'avons dit, ces modèles nationaux sont concurrencés depuis le début des années 1990 par les modèles en données de panel. Faire de la prévision avec de tels modèles fait apparaître une difficulté : celle du passage des prévisions locales à la prévision nationale. Les principaux modèles ont contourné ce problème en établissant directement des prévisions en sièges. En effet, une fois calculées les prévisions locales en sièges, il suffit de sommer ces prévisions pour obtenir la prévision nationale.

Les seuls modèles en données de panel proposant une prévision nationale en voix (ex post ou ex ante) sont ceux de Dubois [2002], Lafay [2002] ${ }^{36}$, Auberger et Dubois [2003, 2005] et Auberger [2005]. Dubois [2002] a été le premier à montrer que la moyenne arithmétique des prévisions locales pouvait tout à fait constituer une prévision nationale

\footnotetext{
${ }^{32}$ On notera une erreur dans le calcul de la prévision du modèle de Lewis-Beck [1997b] qui utilise une croissance du PIB de 3,2\% pour obtenir sa prévision de $51 \%$ pour le candidat de gauche. Mais ce chiffre correspond à une croissance nominale alors que le modèle utilise une croissance réelle. Le chiffre de la croissance réelle était de 1,83\% et donc une prévision de 49,5\% pour le candidat de gauche. Cette erreur est préjudiciable car elle a contraint l'auteur à modifier son modèle alors qu'il n'y avait pas lieu.

$3344,0 \%$ pour la gauche selon l'IFOP, 45,0 \% selon la SOFRES, 46,5\% selon BVA.

${ }^{34}$ Libération du 23/05/1997.

${ }^{35}$ Voir Libération du 21/03/2002.

${ }^{36}$ Pour cette contribution, il s'agit en fait d'une utilisation du modèle de Fauvelle-Aymar et al. [2000].
} 
statistiquement rigoureuse. Toutes les contributions subséquentes ont repris cette méthode à l'exception de Lafay [2002] pour laquelle le passage des prévisions locales à la prévision nationale n'est pas explicité et celle d'Auberger [2005] où la moyenne des prévisions départementales pondérée par le poids électoral de chaque département est calculée.

Du point de vue des sièges, les premières prévisions ex ante diffusées ont été celles du modèle de Jérôme et al. [1993] $]^{37}$. Le modèle prévoyait 453 sièges pour la droite en métropole qui en a en fait obtenu 470. Ce modèle a ensuite été utilisé pour prévoir les résultats des élections législatives de 1997 et il s'est une fois encore très bien comporté avec une prévision (non diffusée) de 242 sièges pour la droite, soit une erreur de 13 sièges seulement (elle en a obtenu en fait 255$)^{38}$. Cependant, ce modèle n'a pas été mis à l'épreuve lors des élections législatives de 2002. Cela provient sans doute du fait que deux des auteurs du modèle de Jérôme et al. [1993] ont créé un nouveau modèle. Ce dernier a connu plusieurs versions (Jérôme et al. [1999, 2003], Jérôme et Jérôme-Speziari [2004]). Lors des élections législatives de 2002, la dernière prévision publiée donnait 129 sièges à la gauche qui en a obtenu en fait 171 soit une erreur considérable de plus de 40 sièges ${ }^{39}$. Enfin, le dernier modèle utilisant des données de panel pour prévoir le résultat des élections législatives au niveau national est celui d'Auberger et Dubois [2003, 2005]. Avant le scrutin, deux prévisions en sièges pour les élections législatives de 2002 avaient été diffusées via Internet $^{40}$ : 172 et 178 sièges pour la gauche $^{41}$. A titre de comparaison, les instituts de sondages donnaient 154 sièges pour CSA, 155 pour la SOFRES et 167 pour l'IPSOS ${ }^{42}$.

Si les élections législatives et présidentielles sont celles qui ont donné lieu à l'élaboration du plus grand nombre de prévisions, on voit émerger depuis la fin des années 1990 des prévisions ex ante pour des élections de second plan : les élections cantonales de 1998 (Le Figaro du 27/02/1998), les élections européennes de 1999 (Le Figaro du 04/06/1999), les élections municipales de 2001 (Jérôme et Jérôme-Speziari [2001] et Le

\footnotetext{
${ }^{37}$ Voir Le Figaro du 19/03/1993 et Lafay [1995a].

${ }^{38}$ Lafay [1998].

${ }^{39}$ La source est un article publié sur le site Internet de L'expansion le 24/05/2002. Pour d'autres prévisions concernant les échéances de 2002, voir également les articles de presse suivants : Les échos du 17/04/2001, Libération du 23/08/2001, La lettre de l'expansion du 24/09/2001, L'expansion du 08/11/2001 et La lettre de l'expansion du 25/03/2002.

40 www.previsions-elections.com

${ }_{41}$ Précisons que ces prévisions étaient issues d'équations de vote et de sièges estimées par les MCO. En tenant compte du fait que l'ensemble formé par ces deux équations représentait un système et en appliquant donc une méthode adéquate (FIML en l'espèce), la prévision aurait été parfaite avec 171 sièges (Auberger et Dubois [2005]). Notons toutefois des erreurs élevées au niveau des départements qui peuvent laisser penser à des compensations d'erreurs.

${ }^{42}$ Il s'agit dans chaque cas du point central d'une fourchette.
} 
Figaro économie du 23/02/2001 ${ }^{43}$ ) les élections européennes de 2004 (Jérôme-Speziari et Jérôme [2003]) et les élections régionales de 2004 (L'expansion du 01/04/2003 et du 11/02/2004 et Libération du 15/03/2004. Aucun modèle n'a cependant donné de résultats véritablement satisfaisants.

Globalement, on constate donc que les élections les plus anciennement étudiées, présidentielles et législatives, sont les mieux prévues et que, parmi les modèles prédisant le résultat de ces élections, ceux présentant l'économétrie la plus rigoureuse obtiennent les meilleurs performances.

\section{Conclusion}

Après 30 années et près de 70 contributions, que ressort-il des études sur le vote en France ?

Tout d'abord les principaux enjeux théoriques et empiriques semblent tranchés. Les modèles utilisent dans leur très grande majorité la théorie du vote rétrospectif et les variables explicatives clés ont été identifiées (croissance du PIB, chômage, popularité, résultat des élections précédentes) tout comme leur délai d'impact (environ un an) même si des interrogations subsistent sur la pertinence des variables économiques subjectives. Concernant la variable dépendante, un relatif consensus existe maintenant quant au choix du sortant. Il reste cependant à mener une réflexion approfondie sur le tour à retenir dans le cas d'élections à deux tours. On peut également penser qu'il serait intéressant de construire des fonctions de vote par parti même si, comme nous l'avons mentionné, quelques études existent déjà.

Du point de vue des élections étudiées et des données utilisées, il manque un modèle législatif en données de panel par circonscription. La construction d'un tel modèle constitue sans nul doute l'un des principaux défis des années à venir. Outre la plus grande finesse qu'il offrirait au regard des prévisions (à la lecture de prévisions régionales ou départementales, on ne sait pas quelles circonscriptions vont basculer), il permettrait peut-être de résoudre l'un des problèmes majeurs qui se pose à la prévision électorale, à savoir le passage des voix aux sièges. A ce propos, la relation entre voix et sièges reste mystérieuse et sa modélisation devra faire l'objet d'améliorations conséquentes dans le futur. De même, toujours sur le plan des élections étudiées, les déterminants des élections cantonales sont encore méconnus dans le

\footnotetext{
${ }^{43}$ A noter que pour ces élections, en dehors des équations de vote et des sondages d'intentions de vote, une troisième méthode de prévision a été expérimentée. Il s'agissait d'un concours de pronostic dont les résultats sont synthétisés dans Foucault et al. [2001].
} 
cadre d'un modèle en données de panel. Ces dernières sont pourtant appelées à prendre une plus grande importance à l'avenir en raison d'une décentralisation toujours croissante.

Ensuite, des progrès restent à faire dans l'économétrie employée. Les principaux tests de validité sont largement négligés. Les chercheurs semblent en effet peu soucieux des grands problèmes économétriques. De même, le caractère de système des fonctions de vote et de sièges n'est que rarement pris en compte. Par ailleurs, la stationnarité et la cointégration dans les panels n'ont jamais été, à notre connaissance, envisagées. La prise en compte d'interactions spatiales, très en vogue actuellement, semble également prometteuse dans le cadre du vote.

Par ailleurs, la modélisation du vote peut être affectée par des modifications du comportement électoral alors même que les équations de vote supposent justement une certaine stabilité. Par exemple, comme nous l'avons dit, les électeurs se décident de plus en plus tard. Cependant, pour ce cas précis, que la décision de vote se prenne la veille du scrutin ou six mois avant, les fonctions de vote conservent leur objectif premier : expliquer le vote. Du point de vue de la structure des équations de vote, on peut penser que ce qui importe vraiment, c'est la stabilité dans les déterminants : les électeurs doivent se décider au regard des mêmes variables d'une élection à l'autre. Maintenant, on pourrait imaginer un modèle adaptatif où les variables économiques seraient définies avec un retard variable par rapport à la date du scrutin mais cela poserait un problème si le modèle est utilisé à des fins de prévision (quel sera le retard à la prochaine élection ?). S'il y a une perte d'efficacité dans le fait que les électeurs se décident tardivement, c'est peut-être précisément sur le plan de la prévision. En effet, lorsque les électeurs se décidaient longtemps en avance, les fonctions de vote étaient construites en conséquence et généraient des prévisions bien avant le scrutin. De nos jours, les retards sont faibles et seules les données politiques et économiques les plus récentes sont pertinentes. Cela contraint les modélisateurs à présenter leurs prévisions peu de temps avant le scrutin ${ }^{44}$. Malgré cela, la prévision à partir de fonctions de vote garde un intérêt qualitatif, celui d'apporter la prévision la plus précise possible même si elle intervient au dernier moment. Par exemple, la veille du premier tour des élections législatives de 2002, il était intéressant non pas de savoir que la droite allait gagner ces élections (ce qui était acquis par le jeu des élections de confirmation) mais de connaître l'ampleur de cette victoire. Comme nous l'avons vu, une équation de vote a d'ailleurs supplanté tous les instituts de

\footnotetext{
${ }^{44}$ La prévision longtemps à l'avance est toujours possible sur la base des valeurs anticipées mais l'absence de ces valeurs pour les données locales, très utilisées, rend cet exercice difficile sinon impossible. Par ailleurs, l'utilisation des valeurs anticipées est un facteur supplémentaire d'incertitude.
} 
sondages en termes de précision, montrant par là même tout l'intérêt de cette méthode de prévision.

2007 et 2008 s'annoncent cruciales avec pas moins de quatre échéances électorales en douze mois qui permettront de juger de la fiabilité des fonctions de vote tant sur le plan explicatif que prédictif et d'établir ainsi leur degré de maturité ${ }^{45}$.

\section{Références bibliographiques :}

AUBERGER A. [2001], Popularité, cycles et politique économique, thèse, Université de Paris 2.

AUBERGER A. [2004], Les fonctions de vote : un survol de la littérature, L'actualité économique, Vol. 80, p. 95-107.

AUBERGER A. [2005], Forecasts of the 2004 French European Election, Swiss Political Science Review, Vol. 11, p. 61-78.

AUBERGER A. et DUBOIS E. [2003], Situation politico-économique et résultats des élections législatives françaises, Revue économique, Vol. 53, p. 551-560.

AUBERGER A. et DUBOIS E. [2005], The Influence of Local and National Economic Conditions on French Legislative Elections, Public Choice, Vol. 125, p. 363-383.

BELANGER E. [2004], Finding and Using Empirical Data for Vote and Popularity Functions in France, French Politics, Vol. 2, p. 235-244.

BELANGER E. et LEWIS-BECK M.S. [2004], National Economic Voting in France: Objective vs Subjective Measures, in Lewis-Beck M.S. [ed], The French Voter, Before and After the 2002 Elections, Palgrave McMillan, p. 231-242.

BON F. et CHEYLAN J.-P. [1988], La France qui vote, Hachette, Paris.

BONNETAIN P. [2004], Behind the Polling-Booth Curtain and Beyond Simple Speculations: Toward a Causal Model of Far-Right Voting Behaviour - Some Evidence from French Presidential Elections of 2002, Canadian Journal of Political Science, Vol. 37, p. 419-429.

CAYROL R., JAFFRE J., MISSIKA J.-L., GIACOMETTI P., LE GALL G. et PARISOT L. [1997], Le modèle économique électoral de l'Iowa : fiabilité, limites et complémentarité, Revue politique et parlementaire, ${ }^{\circ} 989$, p. 65-69.

CAMPBELL A., CONVERSE P.E., MILLER W.E. et STOKES D.E. [1960], The American Voter, John Wiley \& Sons, New York.

\footnotetext{
${ }^{45}$ Voir les premières prévisions dans L'expansion d'avril 2006.
} 
COURBIS R. [1995], De la modélisation macro-économique à la modélisation macropolitique : propos d'étape, Journal de la société de statistique de Paris, Vol. 136, p. 47-70.

DEFFAINS B., JEROME B. et SPEZIARI V. [1996], Décentralisation et compétition fiscale : les limites à la dynamique concurrentielle des territoires, in Brot J. [ed], Entreprise, région et développement, mélanges en l'honneur de René Gendarme, Editions Serpenoise, p. 155-172. DOWNS A. [1957a], An Economic Theory of Political Action in Democracy, Journal of Political Economy, Vol. 65, p. 135-150.

DOWNS A. [1957b], An Economic Theory of Democracy, Harper \& Row, New York.

DUBOIS E. [2001], La prévision électorale en France : où en est-on ?, miméo, LAEP, Université de Paris 1.

DUBOIS E. [2002], Un modèle de prévision par département pour les élections présidentielles françaises, miméo, LAEP, Université de Paris 1.

DUBOIS E. [2005], Économie politique et prévision conjoncturelle : construction d'un modèle macroéconométrique avec prise en compte des facteurs politiques, thèse, Université de Paris 1.

DUBOIS E. et FAUVELLE-AYMAR C. [2004], Vote functions in France and the 2002 election forecast, in Lewis-Beck M.S. [ed], The French Voters: Before and After the 2002 election, Palgrave Macmillan, p. 205-230.

EDGEWORTH F.Y. [1898], Miscellaneous Applications of the Calculus of Probabilities, Journal of the Royal Statistical Society, Vol. 61, p. 534-544.

EGAL Y., FRANCK R., GERTCHEV N., JEROME B., LEMENNICIER B. et VAILLANT N. [2003], Les déterminants politico-économiques du vote vert : une application aux municipales de 2001 et aux présidentielles de 2002 pour la France suburbaine, miméo, LEP, Université de Paris 2.

FAUVELLE-AYMAR C. et LEWIS-BECK M.S. [2005], Coalition Strategies and the National Front Vote in French Legislative Contests, French Politics, Vol. 3, p. 164-177.

FAUVELLE-AYMAR C., LAFAY J.-D. et SERVAIS M. [2000], The Impact of Turnout on Electoral Choices: An Econometric Analysis of the French Case, Electoral Studies, Vol. 19, p. 393-412.

FIORINA M.P. [1981], Retrospective Voting in American National Elections, Yale University Press.

FOUCAULT M. et FRANCOIS A. [2005], Le rendement des dépenses électorales en France, Revue économique, Vol. 56, p. 1125-1143. 
GOODHART C.A.E. et BHANSALI R.J. [1970], Political Economy, Political Studies, Vol. 18, p. 43-106.

HOTELLING H. [1929], Stability in Competition, Economic Journal, Vol. 39, p. 41-57.

JÉRÔME B. et JÉRÔME-SPEZIARI V. [2000], The 1998 French Regional Elections: Why So Much Political Instability?, Electoral Studies, Vol. 19, p. 219-236.

JEROME B. et JEROME-SPEZIARI V. [2002], Les municipales de mars 2001 : vote récompense ou vote sanction?, Revue française de science politique, Vol. 52, p. 251-273.

JÉRÔME B. et JÉRÔME-SPEZIARI V. [2003], A Le Pen Vote Function for the 2002 Presidential Election: A Way to Reduce Uncertainty, French Politics, Vol. 1, p. 247-251.

JÉRÔME B. et JÉRÔME-SPEZIARI V. [2004], Forecasting the 2002 Elections: Lessons from a Political Economy Model, in Lewis-Beck M.S. [ed], The French Voters: Before and After the 2002 election, Palgrave Macmillan, p. 178-204.

JÉRÔME B., JÉRÔME-SPEZIARI V. et LEWIS-BECK M.S. [1999], Polls Fails in France: Forecasts of the 1997 Legislative Election, International Journal of Forecasting, Vol. 15, p. 163-174.

JEROME B., JEROME-SPEZIARI V. et LEWIS-BECK M.S. [2001], Evaluation économique et vote en France et en Allemagne, in Cautrès B. et Reynié D. [eds], L'opinion européenne, Presses de Sciences Po, Fondation Robert Schuman, Paris, p. 101-122.

JÉRÔME B., JÉRÔME-SPEZIARI V. et LEWIS-BECK M.S. [2003], Reordering the French Election Calendar: Forecasting the Consequences for 2002, European Journal of Political Research, Vol. 42, p. 425-440.

JEROME B., LAFAY J.-D. et LEWIS-BECK M.S. [1993], Elections législatives de mars 1993 : prévisions politico-économiques par région, miméo LAEP, Université de Paris 1.

JÉRÔME B. et LEWIS-BECK M.S. [1999], Is Local Politics Local? French Evidence, European Journal of Political Research, Vol. 35, p. 181-197.

JÉRÔME B. et VAILLANT N.G. [2005], The French Rejection of the European Constitution: An Empirical Analysis, European Journal of Political Economy, Vol. 21, p. 1085-1092.

JÉRÔME-SPEZIARI V. et JÉRÔME B. [2003], A Global European Vote Function for the Socialist Parties: Application and Forecast for the 2004 European Elections, Miméo.

KEY V.O. [1966], The Responsible Electorate: Rationality in Presidential Voting, Harvard University Press, Cambridge, MA.

KINDER D.R. et KIEWIET D.R. [1979], Economic Discontent and Political Behavior, the Role of Personal Grievances and Collective Economic Judgements in Congressional Voting, American Journal of Political Science Vol. 23, p. 495-527. 
KRAMER G.H. [1971], Short-Term Fluctuations in U.S. Voting Behavior, 1896-1964, American Political Science Review, Vol. 65, p. 131-143.

KUKLINSKI J. et WEST D. [1981], Economic Expectations and Voting Behavior in United States House and Senate Elections, American Political Science Review, Vol. 75, p. 436-447.

LAFAY J.-D. [1995a], Les interactions entre économie et politique, Journal de la Société de Statistique de Paris, Vol. 136, p. 17-28.

LAFAY J.-D. [1995b], Note sur l'élection présidentielle de 1995 et les apports de l'analyse économétrique des comportements électoraux, miméo, LAEP, Université de Paris 1.

LAFAY J.-D. [1998], Les interactions entre économie et politique. Actes du colloque "La régulation des marchés : quelles leçons pour la Russie en transition", Haut Collège d'Économie, Moscou, p. 37-51.

LAFAY J.-D. [2002], Analyse économique d'une présidentielle, Sociétal, n³6, p. 4-8.

LAFAY J.-D. et JEROME B. [1991], Qualité de la gestion municipale et résultats électoraux des maires sortants : analyse empirique des élections de mars 1989, Économie (Université de Perpignan), p. 35-50.

LAFAY J.-D. et SERVAIS M. [2000], The Influence of Political Scandals on Popularity and Votes, in Lewis-Beck M.S. [ed], How France Votes, Chatham House Publishers, New-York et Londres, p. 189-205.

LAFAY J.-D. et SERVAIS M. [2002], The Impact of Political Scandals on Popularity and Votes, Homo Oeconomicus, Vol. 19, p. 153-170.

LANCELOT A. [1998], Les élections nationales sous la Ve République, P.U.F., Paris.

LAZARSFELD P.F., BERELSON B. et GAUDET H. [1944], The People's Choice, Columbia University Press, New York.

LECAILLON J. [1980], La crise et l'alternance, Cujas, Paris.

LEWIS-BECK M.S. [1983], Economics and the French Voter: A Microanalysis, Public Opinion Quarterly Vol. 47, p. 347-360.

LEWIS-BECK M.S. [1985], Un modèle de prévision des élections législatives françaises, Revue française de science politique, Vol. 35, p. 1080-1089.

LEWIS-BECK M.S. [1986a], Comparative Economic Voting: Britain, France, Germany, Italy, American Journal of Political Science Vol. 30, p. 315-346.

LEWIS-BECK M.S. [1986b], Les législatives de 1986 : nouveau clivage ou restauration ?, Politique Économique, $\mathrm{n}^{\circ} 3$ (juin), p. 31-33.

LEWIS-BECK M.S. [1988], Economics and Elections, the Major Western Democracies, The University of Michigan Press, Ann Arbor. 
LEWIS-BECK M.S. [1991], French National Elections: Political Economic Forecasts, European Journal of Political Economy, Vol. 7, p. 487-496.

LEWIS-BECK M.S. [1995], Comparaison de prévision des élections présidentielles en France et aux États-Unis, Journal de la Société de Statistique de Paris, Vol. 136, p. 29-46.

LEWIS-BECK M.S. [1996], Cross-National Election Surveys: A French Pre-test, Electoral Studies Vol. 15, p. 513-528.

LEWIS-BECK M.S. [1997a], Le vote du "porte-monnaie" en question, in Boy, D. et Mayer, N. [eds], L'électeur a ses raisons, Presses de Sciences Po, Paris, p. 239-261.

LEWIS-BECK M.S. [1997b], Who's the Chef? Economic Voting under a Dual Executive, European Journal of Political Research, Vol. 31, p. 315-325.

LEWIS-BECK M.S. [2000], The Enduring French Voter, in Lewis-Beck M.S. [ed], How France Votes, Chatham House Publishers, New-York et Londres, p. 1-13.

LEWIS-BECK M.S. [2001], Modèles économétriques, in Perrineau P. et Reynié D. [eds], Dictionnaire du vote, P.U.F., Paris, p. 644-646.

LEWIS-BECK M.S. [2005], Election Forecasting: Principles and Practice, British Journal of Politics and International Relations, Vol. 7, p. 145-164.

LEWIS-BECK M.S. [2006], Does Economics Still Matter? Econometrics and the Vote, Journal of Politics, Vol. 68, p. 208-212.

LEWIS-BECK M.S. et BELLUCCI P. [1982], Economic Influences on Legislative Elections in Multiparty Systems: France and Italy, Political Behavior, Vol. 4, p. 93-107.

LEWIS-BECK M.S. et NADEAU R. [2004], Dual Governance and Economic Voting: France and the United States, in Lewis-Beck M.S. [ed], The French Voter, Before and After the 2002 Elections, Palgrave McMillan, p. 136-154.

LEWIS-BECK M.S. et RICE T.W. [1984], Forecasting Presidential Elections: A Comparison of Naive Models, Political Behavior, Vol. 6, p. 9-21.

LEWIS-BECK M.S. et RICE T.W. [1992], Forecasting Elections, CQ Press.

LEWIS-BECK M.S. et STEGMAIER M. [2000], Economic Determinants of Electoral Outcomes, Annual Review of Political Science, p. 183-219.

LEWIS-BECK M.S. et STEGMAIER M. [2006], Economic Models of Voting, in Dalton R. et Klingemann, H.D. [eds.], Oxford Handbook of Political Behavior, Oxford University Press, Oxford, à paraître.

MELTZER A.H. et VELLRATH M. [1975], The Effects of Economic Policies on Votes for the Presidency: Some Evidence from Recent Elections, Journal of Laws and Economics, Vol. 18, p. 781-805. 
MUELLER J.E. [1970], Presidential Popularity from Truman to Johnson, American Political Science Review, Vol. 64, p. 18-35.

NORPOTH H. [1996], The Economy, in Leduc L., Niemi R.G. et Norris P. [eds], Comparing Democracies: Elections and Voting in Global Perspective, Sage Publications, p. 299-318.

PALDA F. et PALDA K. [1998], The Impact of Campaign Expenditures on Political Competition in the French Legislative Elections of 1993, Public Choice, Vol. 94, p. 157-174.

PALDAM M. et NANNESTAD P. [2000], What Do Voters Know About the Economy? A Study of Danish Data, 1990-1993, Electoral Studies, Vol. 19, p. 363-391.

POPKIN S.L. [1991], The Reasoning Voter, University of Chicago Press.

ROSA J.-J. [1980], Economic Conditions and Elections in France, in Whiteley P. [ed], Models of Political Economy, Sage Modern Politics Series vol. 4, Sage Publications, Londres \& Beverly Hills, p. 101-120.

ROSA J.-J. et AMSON D. [1976], Conditions économiques et élections, Revue française de science politique, Vol. 25, p. 1101-1124.

SERVAIS M. [1997], Les modèles économétriques du vote, in Mayer N. [ed], Les modèles explicatifs du vote, L'Harmattan, Paris, p. 133-153.

THEIL H. [1970], The Cube Law Revisited, Journal of the American Statistical Association Vol. 65, p. 1213-1219.

WEATHERFORD M.S. [1978], Economic Conditions and Electoral Outcomes: Class Differences in the Political Response to Recession, American Journal of Political Science Vol. 22, p. 917-938. 